\title{
A Study of Efficient Speaklet Driving Mechanisms for Use in a Digital Loudspeaker Array Based on PZT Actuators
}

\author{
Sangchai Monkronthong, Neil White, Nick Harris \\ Electronics and Computer Science \\ University of Southampton \\ Southampton, UK \\ sm12e11,nmw,nrh@soton.ac.uk,
}

\begin{abstract}
The concept of a Digital Loudspeaker Array (DLA) requires a driving signal for a speaklet (tiny loudspeaker) with a short rectangle pulse. The speaklet takes the form of a diaphragm, which vibrates freely and emits acoustic energy at its resonant frequency. This study gives a guideline for the design of PZT speaklets for assigning the required resonant frequency and maximizing the amplitude of the acoustic response. The speaklet consists of three layers; a diaphragm, an active PZT layer, and a bottom electrode. The device is simulated with Comsol Multiphysics. From the simulation, the first resonant frequency mainly depends on diaphragm diameter and thickness while the maximum displacement is related to thickness of the three layer structure. Additionally, this study verifies the concept of a multiple-level DLA that can produce an acoustic signal with a linear relationship between its amplitude and the driving pulse width. This result allows a more flexible design of DLA as the limitation of one bit of resolution per speaklet is removed, allowing designs with a reduced number of speaklets.
\end{abstract}

Keywords—digital loudspeaker array,PZT actuator

\section{INTRODUCTION}

A Digital Loudspeaker Array (DLA) is a loudspeaker consisting of a number of tiny loudspeakers, which are called speaklets. These are driven with a train of constant-width rectangular pulses in order to reconstruct audible sound [1]. When a rectangular pulse is fed to a speaklet, it will ideally produce a short acoustic pulse, called a 'click'. The loudness of the sound produced depends on the number of speaklets emitting clicks [1]. The DLA will produce the desired sound waveform depending on the uniformity and linearity of the clicks of all the speaklets emitting within the array. It is important to make all speaklets within the DLA identical, which is a key requirement for sound reconstruction. In such a system the effective bit resolution is given by the number of speaklets. Manufacturing such systems is difficult, but planar technologies such as thin film or even thick film are candidates as it is suitable for making numerous, small and identical structures. For example, Dejaeger et al and Casset et al [2][3]. developed a DLA with 256 Lead Zirconate Titanate (PZT) based speaklets.
In our earlier work [4], we proposed the concept of a Multiple-Level Digital Loudspeaker Array (MDLA). This addressed the practical issue of the sheer number of speaklets required for high resolution sound reproduction, by increasing the number of quantization levels of acoustic output for a single speaklet by using a range of different pulse widths, which linearly relate to maximum acoustic response. Speaklets with ideal acoustic response for digital sound reconstruction were simulated in order to verify the concept theoretically. In this paper, we will describe the relationship of the speaklet structure and its surface vibration response to a digital pulse and simulate the effect with Comsol. In addition, the concept of the MDLA is verified experimentally. Together these results inform the design parameters of MDLA speaklets, and future work will concentrate on utilizing these results to implement a DLA.

\section{MoDELLING}

\section{A. Finite Element Model (FEM):}

Speaklets were modelled in Comsol Multiphysics in order to study surface vibration response to a unit step pulse. This pulse had an amplitude of $5 \mathrm{~V}$, and a 40 ns rise time. In order to study maximum displacement of the speaklet diaphragm with different sizes, which vibrate with different natural frequencies, a unit step is used rather than a retangular pulse. Owing to the width of the electrical pulse corresponsing to the frequency of the oscillation, a unit step allows a diaphragm to oscillate with the maximized displacement. The structure of the speaklet is based on a typical piezo-buzzer, which has a round disk as a diaphragm fixed around the edge. The diameters of the PZT and the bottom electrode are equal, as shown in figure $1 \mathrm{a}$.

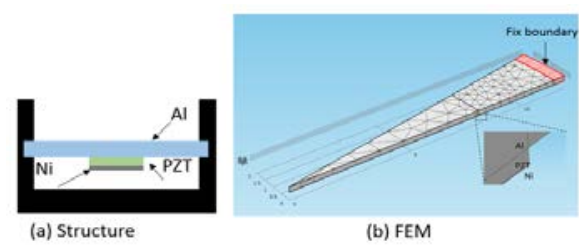

Figure 1: PZT speaklet cross section schematic view and FEM model 
TABLE 1: SPEAKLET COMPONENTS AND THEIR CHARACTERISTICS
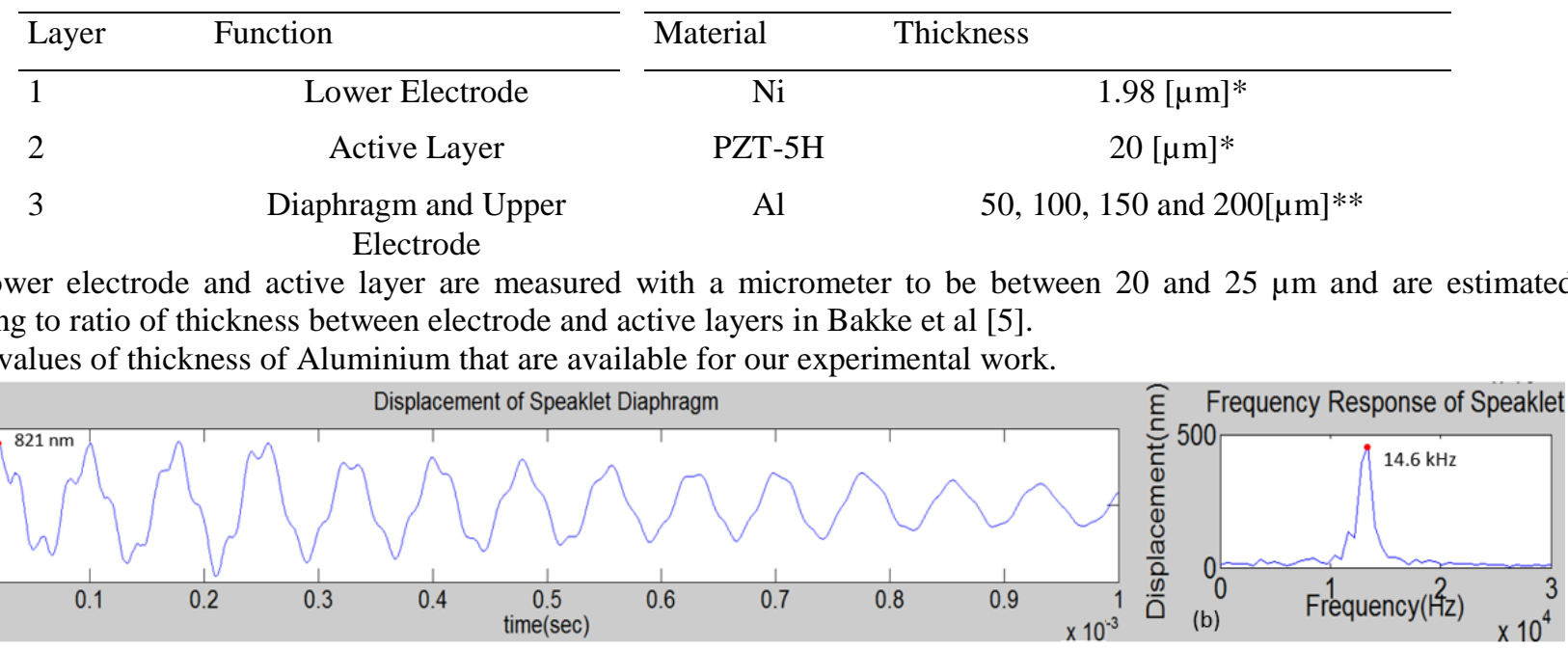

Figure 2: Speaklet displacement and frequency response with diaphragm $12 \mathrm{~mm}$ diameter, $0.2 \mathrm{~mm}$ thickness and electrode diameter of 9.1 $\mathrm{mm}$.

For the purpose of undertaking a FEM, a segment of $1 / 10$ of the disk is simulated in order to reduce the simulation time and this is shown in Figure 1b. The other parameters used are shown in Table 1

\section{Characterization of Diaphragm Vibration Response:}

The FEM study of the membrane's response to an unit step allows the extraction of two important parameters for the DLA design. These are the fundamental resonant frequency $\left(\mathrm{f}_{0}\right)$, and the displacement amplitude of the first pulse of the response. Figure 2 shows an example response. Figure $2 \mathrm{~b}$ is the frequency domain of the displacement response shown in Figure 2a. These parameters will affect the allowable driving pulse rate of the DLA and also the loudness of the reconstructed sound. For the following study, the diameter and thickness of the diaphragm and lower electrode are chosen as variable parameters.

The FEM results are divided into four categories as we vary the;

- $\quad$ radius of the diaphragm,

- radius of the electrode,

- thickness of the electrode

- thickness of the PZT layer

A summary of these results are shown in graphs a, b, c and d respectively in Figure 3. The top row shows the effect on resonant frequency and the second row shows the effect on displacement. The individual curves in the graphs represent different thickness of diaphragm, as per the legends. For the study of the effect of the dimensions of the diaphragm, the lower electrode radius was fixed at $0.5 \mathrm{~mm}$ in order that all speaklets with diaphragm radius from 1 to $6 \mathrm{~mm}$ are exerted with the same force from the area of electrode. It can be seen that when reducing the diameter of the diaphragm or increasing its thickness, the resonant frequency increases (as shown in figure 3a.1. This is expected as equation 1 shows the classical relationship between thickness (h), diameter (D) and resonant frequency $\left(f_{r}\right)$ of a diaphragm, showing a linear relationship with thickness but a $1 / \mathrm{D}^{2}$ relationship with diameter.

$$
\cdot f_{r}=\frac{1.642 h}{D^{2}} \sqrt{\frac{Y}{\rho\left(1-\left(\sigma^{E}\right)^{2}\right)}} \cdot
$$

In equation $1, Y$ is Young's modulus, $\rho$ is density and $\sigma^{\mathrm{E}}$ is Poisson's ratio and are constant [6]. Considering displacement, an increase in thickness of the diaphragm will decrease the displacement while a reduction in diameter of diaphragm decreases the displacement. The slope of the displacement change depends on thickness of diaphragm as shown in Figure 3a.2. When considering a constant dimension of diaphragm but varying only the electrode diameter (as shown in Figure 3b), the relationship between displacement and electrode size is of the form of an inverted parabola having a peak of displacement at approximately three quarters of the diaphragm diameter because a bending moment is generated on the edge of the electrode. The peak of the relationship can be considered as an optimized diameter for the electrode for a diaphragm. In Figure 3b.2, the position of the peak displacement with ratio of electrode to diaphragm diameter varies with the thickness of diaphragm. 

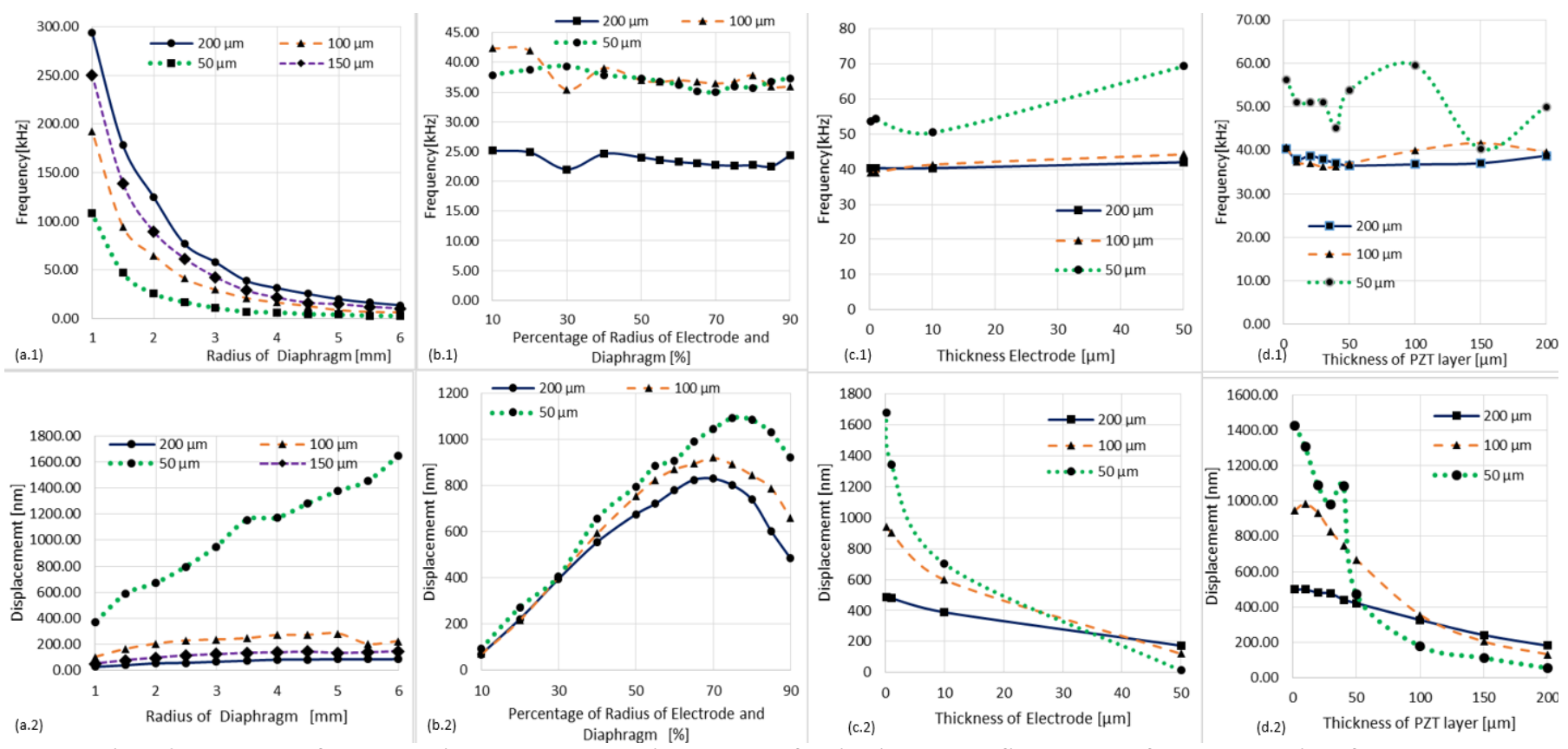

Figure3: The result of the two main parameters: the displacement of oscillation and the first resonant frequency obtained from FEM.

For example, a device with a diaphragm thickness of $50 \mu \mathrm{m}$ has a peak displacement that occurs at an electrode/diaphragm ratio of $75 \%$, but this reduces to about $70 \%$ when the thickness is increased to $200 \mu \mathrm{m}$. However, this ratio has very little effect on the devices resonant frequency, as shown in in Figure 3b.1. Figure 3c shows that an increase in thickness of the bottom electrode has a slight effect on the frequency while an increase in the electrode thickness can reduce the maximum displacement of the speaklet diaphragm. The degree of degradation depends on the thickness of the diaphragm. Similar results are found when the PZT layer is varied. as shown in Figure 3d. A constraint on the thickness of the PZT layer (other than mechanical construction issues) is that the breakdown field strength of the PZT cannot be exceeded, which will be the limiting factor in terms of electrical drive. This is typically of the order of $4 \mathrm{MV} / \mathrm{m}$ for thick film PZT [7] and so a PZT thickness of $50 \mu \mathrm{m}$ can withstand up to $200 \mathrm{~V}$. However, this may be an issue if thin films of the order of a few microns are used, as the voltage would fall to a few volts or 10 s of volts.

Although this FEM study has concentrated on the mechanical dynamics rather than the acoustics, the acoustics follows because the displacement drives the air and they are linked by boundary conditions [8].

\section{EXPERIMENTAL PROCEDURE}

Two experiments in this study are set up according to Figure 4. The pulse generator is implemented with a FPGA board and the digital amplifier is a simple switching circuit. A $12-\mathrm{mm}$ buzzer is used as a speaklet. Its dimensions are the same as FEM speaklet in Figure 2. The first experiment was designed to identify and verify the resonant frequency of the device, and measure its dynamic response.

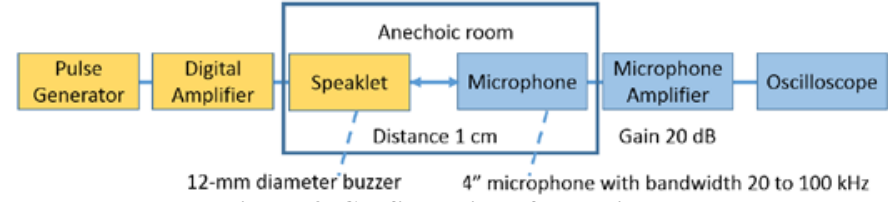

Figure 4: Configuration of Experiment

For this experiment, the speaklet is driven by a pulse with a height of $30 \mathrm{~V}$, a width of $16 \mu$ sec and a repetition pulse rate of $1 \mathrm{kHz}$ or pulse period of $1 \mathrm{~ms}$. With this pulse period, the acoustic pulses should be independent, in that the ringing of the first pulse will have died away before the next pulse is fired. As a result, the response of sequential pulses will be identical and they oscillate with a resonant frequency of 18.5 $\mathrm{kHz}$ as shown in Figure 5. The resonant frequency is different from the result of FEM speaklet $(14.6 \mathrm{kHz})$ because the fixed boundary of the real speaklet is not as perfect as FEM. The error in estimation of thickness of PZT and electrode and air pressure might have effect on the gap of frequencies.

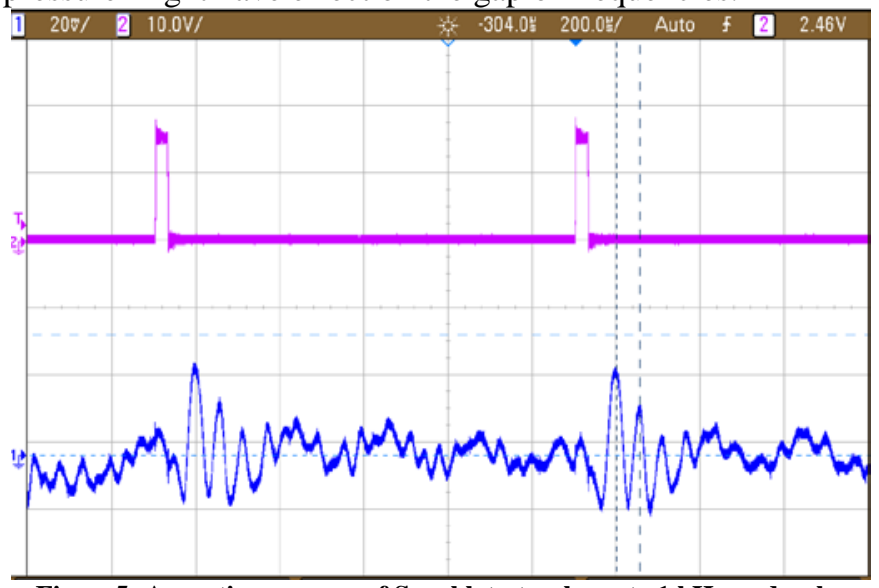

Figure5: Acoustic response of Speaklet at pulse rate $1 \mathrm{kHz}$ and pulse width 16 psec 


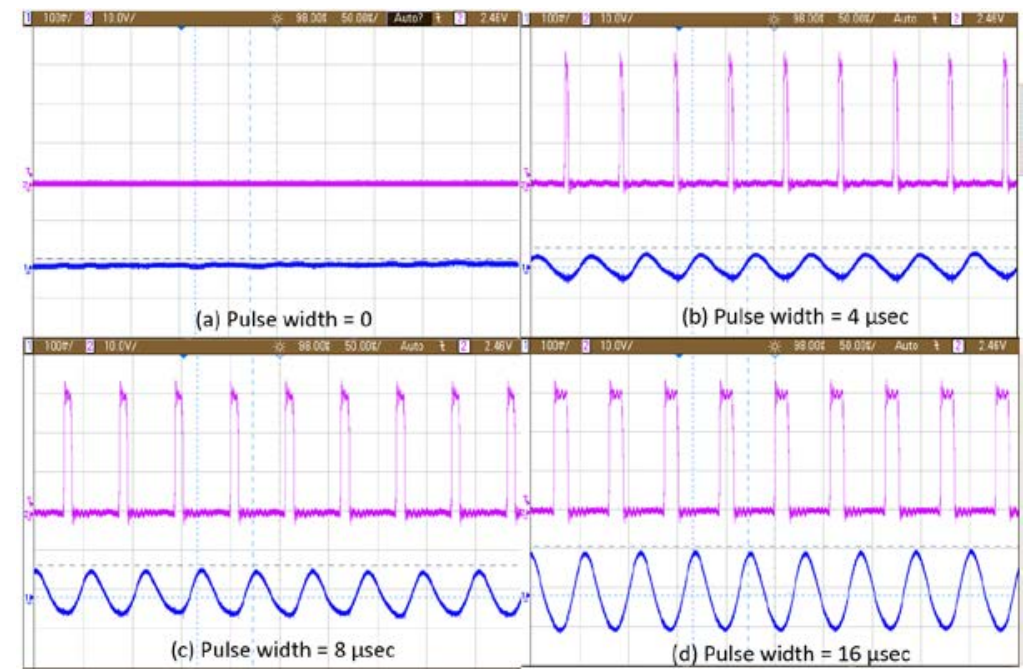

Figure 6: Electrical pulses with different width of $0,4,8$ and $16 \mu$ and their acoustic response

The result is similar to the vibrating response as shown in Figure 2a. There is an underdamped oscillation in this system. This oscillation results from the acoustic mismatch between the diaphragm and air. At the boundary between the two media, there are reflections and transmission of energy with incident energy generated by the PZT layer. Due to the large mismatch, the transmitted energy is far less than the reflected energy. Therefore, the transmitted energy is gradually reduced to zero depending on the number of reflections inside the transducer [9]. This phenomenon is what leads to 'ringing'.

The second experiment is designed to verify that by changing the driving pulse width, the average acoustic amplitude can be altered. For this experiment, the speaklet is driven by a pulse with height of $30 \mathrm{~V}$ and pulse rate of 18.5 $\mathrm{kHz}$, which equals to the resonant frequency of the speaklet. The width of the pulse is varied from zero to $30 \mu$ sec in steps of 2 usec. A microphone with a sensitivity of $4.1 \mathrm{mV} / \mathrm{Pa}$ feeding a 20dB amplifier was positioned at distance of $1 \mathrm{~cm}$ from the speaklet, with the FPGA operating at a master frequency of $100 \mathrm{MHz}$, allowing a pulse resolution of $10 \mathrm{nS}$. Some of the results of the acoustic response are shown in Figure 6 and the amplitude of the acoustic response is plotted together with the pulse width. It can be seen that for pulse widths within the range of 0 to $18 \mu \mathrm{sec}$, the increase in amplitude of the acoustic response is linear as shown Figure 7. The measured relationship has an $\mathrm{R}^{2}$ coefficient of 0.9919 and the speaklet can produce a maximum loudness of $100.3 \mathrm{~dB}$. This result verifies one of the operating principles of the MDLA and shows that with a pulse width resolution of 10 ns for example, 1800 different amplitude levels can be achieved by a single speaklet driven by a pulse.

It must be noted that this acoustic response is different from the acoustic response of the ideal speaklet. The ideal speaklet fed with an electrical pulse produces a well-damped pulse of acoustic response. However, the real device driven with a pulse emits a train of acoustic pulses (ringing).

In order to maintain the first requirement of digital reconstruction [1], which is that pulses must not interfere with each other, the ringing causes a large increase in the period of ending of oscillation. As a result, the pulse rate of DLA is not enough to reconstruct audible sound. Therefore, the ringing needs to be suppressed in order to reduce the unwanted effect. In contrast, if the first requirement of digital reconstruction is omitted and the electrical pulses are transmitted at the resonant frequency, the amplitude of the acoustic pulse will be magnified due to reinforcement of acoustic pulses before it. From the experimental observations, feeding the same pulse width with a different pulse rate, the amplitude of the acoustic response with pulse width of $16 \mu$ sec is improved 5 times from $40 \mathrm{mV}$ at $1 \mathrm{kHz}$ in Figure 5 to $200 \mathrm{mV}$ at $18.5 \mathrm{kHz}$ of $\mathrm{V}_{\mathrm{pp}}$. in Figure 6d. However, feeding pulses at the resonant rate $(18.5 \mathrm{kHz})$ introduces distortion in the digital sound reconstruction due to consecutive pulse interference.

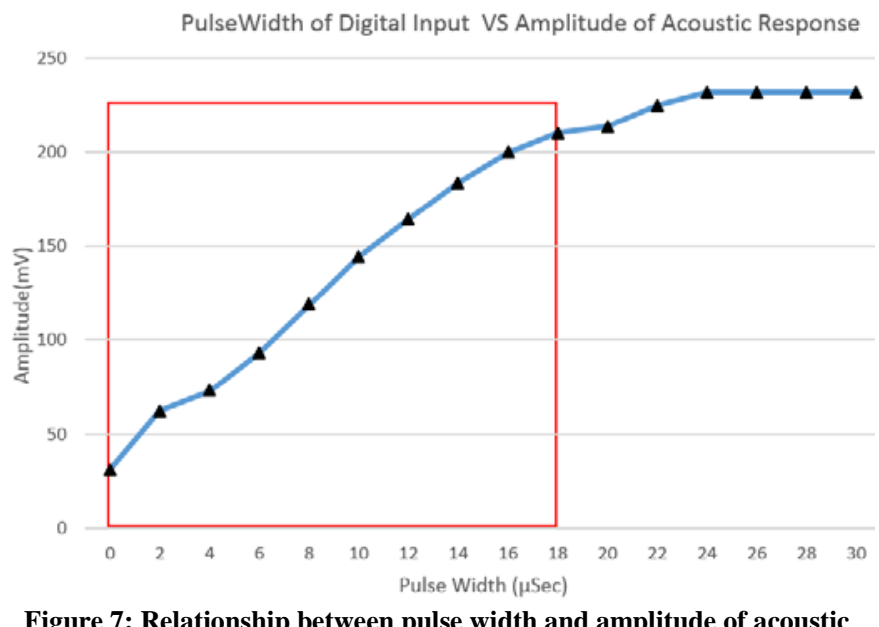

Figure 7: Relationship between pulse width and amplitude of acoustic response

The speaklets for DLA are designed to be single and narrow band as shown in Figure 2b, rather than wide band as with a normal loudspeaker, because the oscillation of the diaphragm is a simple harmonic movement with a single frequency, as shown in Figure 2a. This can be designed by making diaphragms thicker or smaller to have the desired natural frequency. 


\section{CONCLUSIONS AND FUTURE WORKS}

The mechanism of sound reconstruction of DLA relates to trains of pulses feeding speaklets, the natural frequency and the amplitude of vibration of the speaklet diaphragm within the array. The resonant frequency linearly relates to the thickness of diaphragm and hyperbolically with the diameter of diaphragm. The amplitude relates to the thickness and diameter of the diaphragm. The maximum value of the amplitude can be derived from the peak of the invertedparabolic relationship between the size of the electrode and the maximum displacement. The applied voltage is limited by the thickness of the PZT layer althought the thinner the PZT and electrode layer are, the higher the potential amplitude is. For an applied voltage of $10 \mathrm{~V}$, thickness of PZT layer is required to be $2 \mu \mathrm{m}$ in order to produce an electric field strength of 4 $\mathrm{MV} / \mathrm{m}$. A potential enabling technology for the implementation of low voltage speaklets is thin or thick-film, which can produce PZT layers having high values of $d_{33}$, typically being $300 \mathrm{pC} / \mathrm{N}$ estimated from the speaklet in experiment. The thickness of the PZT and electrode layer degrade the amplitude and the degree of the reduction relates to thickness of the diaphragm.

Although most of the results are based on FEM simulation, these allow us to understand the characterization of speaklets and is this is a good approximation in the design for a DLA. The thickness and diameter of diaphragm are the main variables in designing the resonant frequency of acoustic response of a speaklet. The thickness is the key parameter for DLA efficiency because decrease in the thickness makes a speaklet smaller in diameter and higher in vibration amplitude for a given resonant frequency. The reduction in size of a speaklet results in not only an increase in number of speaklets within a certain size of DLA but also expansion in directivity of sound radiation of the speaklet. Increasing the number of speaklets causes improvement in the bit resolution of sound reproduction. Although speaklets can be implemented with thin-film and thick-film technologies, the thin-film technology produces more efficient speaklets, especially for low voltage due to limitation of technologies in production in thickness of layers of a transducer. Screen printing technology can produce speaklets with thickness of PZT and electrode layers in the order of tens of microns, while thin-film technology can produce thickness of an electrode layer in the order of onetenth of a micron and thickness of a PZT layer in the order of a micron.

However, the response of a speaklet to a digital pulse from the FEM simulation is vibration on surface of diaphragm. It is sufficient to study the resonant frequency of the acoustic response because the frequencies of the vibrating surface and acoustic response are the same. In order to the quantify the loudness and directivity of sound, the response has to transform this diaphragm vibration into sound radiation by using the wave equations. Therefore, future work will develop the FEM simulation.
From the pulse response in simulation and experiment 1, there is small difference between FEM and experimental results of the speaklet resonant frequency but the effect of ringing is apparent. Although this causes sound distortion or reduction in pulse rate of speaklet-driving pulses, it may also be possible to use this to increase speaklet loudness when the pulse rate is equal to the speaklet resonant frequency, if high fidelity is not required. Therefore, a study on the ringing effect on sound reconstruction and potential techniques to deal with the resulting distortion is interesting future work.

Experiment 2 shows that the width of rectangle pulse feeding into a speaklet linearly affects the amplitude of acoustic signal from the speaklet for a defined range of widths. This concept results in a significant improvement in bitresolution quality of sound from the original concept of DLA, in which the level of sound depends on the number of speaklets. Using a pulse generator with higher clock speed or using a speaklet with lower resonant frequency (longer period) will improve quality of bit resolution of a speaklet for MDLA.

In addition, this study can be applied in design of the dimensions of diaphragm and transducer of ultrasonic transmitters/sensors for desiring frequency and maximized sensitivity because speaklets can be considered as ultrasonic devices. The resonant frequency of speaklet responses is the transmitting frequency and the amplitude of the response can be implied as the sensitivity of sensor devices. Such arrays may be phase-steerable, opening interesting applications in direction sensitive ultrasonic transmitter arrays.

\section{REFERENCES}

[1] B. M. Diamond, "Digital Sound Reconstruction Using Arrays of SMODMEMS Microspeakers,” 2002.

[2] R. Dejaeger, F. Casset, B. Desloges, G. Le Rhun, P. Robert, S. Fanget, Q. Leclère, K. Ege, and J.-L. Guyader, "Development and Characterization of a Piezoelectrically Actuated MEMS Digital Loudspeaker,” Procedia Eng., vol. 47, pp. 184-187, Jan. 2012

[3] F. Casset, R. Dejaeger, B. Laroche, B. Desloges, Q. Leclere, R. Morisson, Y. Bohard, J. P. Goglio, J. Escato, and S. Fanget, "A 256 MEMS Membrane Digital Loudspeaker Array Based on PZT Actuators," Procedia Eng., vol. 120, pp. 49-52, 2015.

[4] S. Monkronthong, N. M. White, and N. R. Harris, "Multiple-level Digital Loudspeaker Array,” Procedia Eng., vol. 87, pp. 404-407, 2014.

[5] T. Bakke, A. Vogl, O. Żero, F. Tyholdt, I.-R. Johansen, and D. Wang, "A novel ultra-planar, long-stroke and low-voltage piezoelectric micromirror,” J. Micromechanics Microengineering, vol. 20, no. 6, p. 064010, Jun. 2010.

[6] I. Henderson, Piezoelectricx Ceramics: Principle and Application, 2nd ed. APC International, Ltd, 2011.

[7] Harris, N. R., Hill, M., White, N. M., \& Beeby., S. P. (2004). Acoustic power output measurements for thick-film PZT transducers. Electronics Letters, 40(10), 636-637. doi:10.1049/el

[8] M. C. Junger and D. Feit, Sound, Structures and Their Interaction, 2nd ed. The MIT Press, 1972.

[9] F. W. Kremkau, Diagnostic Ultrasound: Principles and Instrument, 6th ed. W.B. SAUNDERS COMPANY, 2006. 